\title{
OBSERVED FREQUENCY VARIATIONS OF SOLAR $p$-MODE TRAVEL TIMES AS EVIDENCE FOR SURFACE EFFECTS IN SUNSPOT SEISMOLOGY
}

\author{
D. C. Braun and A. C. Birch \\ NorthWest Research Associates, CoRA Division, 3380 Mitchell Lane, Boulder, CO 80301; dbraun@cora.nwra.com, aaronb@cora.nwra.com \\ Received 2006 April 18; accepted 2006 July 5; published 2006 August 7
}

\begin{abstract}
Using helioseismic holography, we measure acoustic ( $p$-mode) travel-time perturbations, observed within solar active regions, as functions of frequency and phase speed. We find evidence for a frequency variation, at fixed phase speed, of the travel times that has not previously been reported. This variation is not expected from typical sound-speed models of sunspots, which result from the inversion of travel times and may indicate a significant contribution to the travel times from structures with vertical scales smaller than about $1 \mathrm{Mm}$ near the solar surface.
\end{abstract}

Subject headings: Sun: helioseismology — Sun: interior — Sun: magnetic fields — sunspots

\section{INTRODUCTION}

The discovery two decades ago that sunspots act as both absorbers (Braun et al. 1987) and refractors (Abdelatif et al. 1986) of incident solar acoustic waves ( $p$-modes) offered the promise of probing the subsurface structure of sunspots (Thomas et al. 1982). Models of sunspots as perturbations in the background sound speed have subsequently been constructed using observations from a variety of local helioseismic techniques, including Hankel analysis (Fan et al. 1995), ring diagrams (Basu et al. 2004), and holography (Lindsey \& Braun 2005b). Travel times inferred from time-distance helioseismology have been inverted to model flows and sound-speed perturbations using Fermat's principle and the ray approximation (e.g., Kosovichev \& Duvall 1997; Kosovichev et al. 2000; Zhao et al. 2001; Hughes et al. 2005), the Fresnel-zone approximation (e.g., Jensen et al. 2001; Couvidat et al. 2004), and the Born approximation (e.g., Couvidat et al. 2006).

However, uncertainty exists about the degree to which surface effects may contribute to the observed helioseismic signatures (e.g., Lindsey \& Braun 2005b; Zhao \& Kosovichev 2006). Published three-dimensional (3D) inversions of travel times, to date, do not include any provision for contribution from the near surface layers, which are not resolved by the $p$-mode set used in the observations (the typical vertical resolution near the photosphere is around $1 \mathrm{Mm}$; see Couvidat et al. 2006). Examples of possible surface effects include strong perturbations in the sound speed within about $1 \mathrm{Mm}$ of the photosphere, as suggested by Fan et al. (1995), or contributions to phase shifts due to mode conversion in magnetic fields (e.g., Cally et al. 2003; Crouch et al. 2005). Surface effects resulting from changes in the upper turning points of modes due to magnetic fields have also been proposed (e.g., Kosovichev \& Duvall 1997; Chou et al. 2000; Braun \& Lindsey 2000; Barnes \& Cally 2001). Schunker et al. (2005) report that helioseismic phase shifts obtained from seismic holography in sunspot penumbrae vary with the component of the line-of-sight angle projected into the plane containing the magnetic field and the vertical direction, implying that a significant component of the penumbral phase shifts are photospheric in origin.

The observations used in 3D travel-time inversions are typically made over a single wide frequency bandpass and do not easily allow the assessment of possible frequency-dependent surface terms, analogous to those included in one-dimensional (horizontally invariant) structural inversions performed in global helioseismology (e.g., Christensen-Dalsgaard et al. 1988) and some ring-diagram analyses (e.g., Basu et al. 2004). In addition, the formalism for including possible surface effects (which are likely to be highly variable in the horizontal spatial dimensions) has not been developed for 3D inversions in local helioseismology. A strong motivation for undertaking this potentially substantial task would be observational evidence of frequency variations in observed travel times in magnetic regions that are not consistent with depth structures resolvable by current inversions. Some observations (e.g., Chou et al. 2000; Braun \& Lindsey 2000; Lindsey \& Braun 2004) have suggested frequency variations, but these were not explored in the context of identifying the depth of the sources of the traveltime perturbations. In this letter we report significant frequency variations in $p$-mode travel times in sunspots as measured from helioseismic holography.

\section{OBSERVATIONS AND ANALYSIS}

Helioseismic holography $(\mathrm{HH})$ is a method based on the phase-coherent imaging of the solar interior acoustic field by computationally extrapolating the surface acoustic field into the solar interior (Lindsey \& Braun 1997, 2000). Here HH is performed in the wavenumber-frequency (Fourier) domain (e.g., Lindsey \& Braun 2000), which facilitates the selection of a set of fixed frequency bandpasses with widths of $1 \mathrm{mHz}$ centered at frequencies $\nu_{0}$, of $2,3,4$, and $5 \mathrm{mHz}$. To facilitate comparisons of these observations with time-distance analyses, the analysis in this letter consists of what are termed local control correlations (Lindsey \& Braun 2005a). These are directly comparable to center-annulus time-distance correlations (e.g., Duvall et al. 1996; Braun 1997). In the Fourier domain the $\mathrm{HH}$ control correlations are described by a phase perturbation $\delta \phi$, which is related to an equivalent travel-time perturbation by $\delta \tau=\delta \phi / 2 \pi \nu_{0}$. Here we consider the mean traveltime perturbations (relative to nearby quiet-Sun travel times) determined from and averaged over the ingression and egression control correlations made with a full annular pupil.

A $27 \mathrm{hr}$ duration of full disk Dopplergrams with a 1 minute cadence, obtained from the Michelson Doppler Imager (MDI; Scherrer et al. 1995) on board the Solar and Heliospheric Observatory (SOHO), were used in this study. The data set starts on 2002 April 1, 21:01 UT, and includes several sunspot groups (NOAA ARs $9885,9886,9887$, and 9888 ) within a $60^{\circ}$ by $60^{\circ}$ Postel-projected area. To ensure the relevance of the results to 
TABLE 1

Phase Speed Filter Parameters

\begin{tabular}{ccccr}
\hline \hline Filter & $\begin{array}{c}\text { Pupil Radii } \\
(\mathrm{Mm})\end{array}$ & $\begin{array}{c}w \\
\left(\mathrm{~km} \mathrm{~s}^{-1}\right)\end{array}$ & $\begin{array}{c}\delta w \\
\left(\mathrm{~km} \mathrm{~s}^{-1}\right)\end{array}$ & $\begin{array}{c}\nu_{0} \\
(\mathrm{mHz})\end{array}$ \\
\hline $\mathrm{A} \ldots \ldots$. & $3.5-7.7$ & 12.9 & 1.3 & 5 \\
$\mathrm{~B} \ldots \ldots$. & $7.7-11.8$ & 15.7 & 2.2 & 4,5 \\
$\mathrm{C} \ldots \ldots$. & $11.8-16.0$ & 20.2 & 3.2 & $3,4,5$ \\
$\mathrm{D} \ldots \ldots$. & $16.0-20.2$ & 26.3 & 4.2 & $3,4,5$ \\
$\mathrm{E} \ldots \ldots$. & $20.2-27.1$ & 33.6 & 4.5 & $2,3,4,5$ \\
$\mathrm{~F} \ldots \ldots$. & $27.1-34.1$ & 39.4 & 2.4 & $2,3,4,5$ \\
$\mathrm{G} \ldots \ldots$. & $34.1-41.1$ & 43.5 & 2.5 & $2,3,4,5$ \\
$\mathrm{H} \ldots \ldots$. & $41.1-48.0$ & 48.5 & 3.5 & $2,3,4,5$ \\
$\mathrm{I} \ldots \ldots$. & $48.0-55.0$ & 54.2 & 3.5 & $2,3,4,5$ \\
$\mathrm{~J} \ldots \ldots$. & $55.0-61.9$ & 59.5 & 2.9 & $2,3,4,5$ \\
\hline
\end{tabular}

time-distance analyses and models, we employed narrow annular pupils and corresponding phase-speed filters to the data sets. The phase-speed filters, designated A-J, were of the form prescribed by Couvidat et al. (2006) with central phase speeds $w$, widths $\delta w$, and pupil radii as indicated in Table 1 . The $f$ mode was filtered out, and some filter/frequency combinations were not used due to the failure of any $p$-mode ridge to intersect the filtered domain. The $\mathrm{HH}$ analysis was performed in the eikonal approximation (Lindsey \& Braun 2000), and the data were corrected for small effects due to reduced oscillatory amplitudes in magnetic regions (Rajaguru et al. 2006) by dividing the signal in each pixel by its rms value over the frequency bandpass.

\section{RESULTS}

\subsection{Frequency Variations at Fixed Phase Speed}

Some sample maps of mean travel-time perturbations are shown in Figure 1. In general, there are significant perturbations that appear closely related to the surface magnetic flux density (see § 3.2). Although most maps show travel-time reductions in magnetic regions, there are significant travel-time increases observed with some of the smallest annuli (and phase speeds). What is surprising is the amount of variation among maps made with the same phase speed but different frequencies. For most filters (i.e., D-J) the travel-time perturbations show a general, and substantial, increase in strength with higher frequencies (e.g., Figs. $1 e-1 h$ ). For filters B and C, the travel-time perturbations actually switch sign in sunspot umbrae, from positive values (relative to quiet Sun) at lower frequencies to negative values at higher frequencies (e.g., compare Figs. $1 c$ and $1 d$ ). The distinction appears to be whether the filter/frequency-bandpass combination is dominated by $p_{1}$-modes (resulting in positive travel-time perturbation in sunspot umbrae) or modes with higher radial order (which show negative perturbations throughout active regions).

\subsection{Spatial Relationships with Surface Flux Density}

The travel-time perturbations are nonlinearly related to the surface flux density $B_{\text {tot }}$ and in general exhibit one of three types of behaviors illustrated in Figure 2. The smallest phasespeed filter (A, which is only used at $5 \mathrm{mHz}$ ) is unique in that it shows a positive travel-time perturbation which increases with $B_{\text {tot }}$ (Fig. $2 a$ ). The other two $p_{1}$-dominated filter/frequency combinations (B at $4 \mathrm{mHz}$ and $\mathrm{C}$ at $3 \mathrm{mHz}$ ) show a negative $\delta \tau$, which decreases with increasing $B_{\text {tot }}$, and an abrupt reversal to positive $\delta \tau$ at flux densities typical of sunspot umbrae (e.g., Fig. $2 b$ ). All other combinations show a decreasing $\delta \tau$ (e.g.,

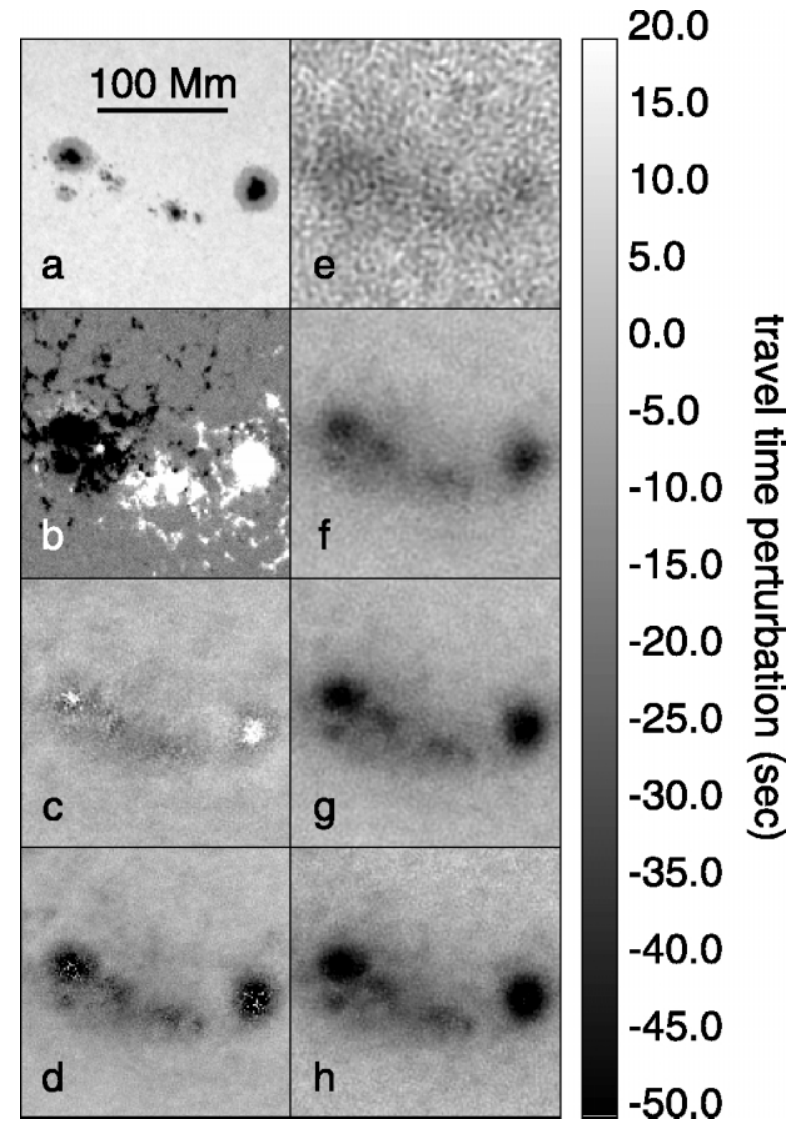

FIG. 1.-(a) MDI intensity, $(b)$ line-of-sight magnetogram, and $(c-h)$ sample maps of the mean travel-time perturbation covering a portion of the region studied and showing sunspot group AR 9885. Panels $c$ and $d$ show travel-time maps for filter $\mathrm{C}$ at 3 and $4 \mathrm{mHz}$, respectively (see Table 1). Panels $e-h$ show the travel-time maps for filter $\mathrm{E}$ at $2,3,4$, and $5 \mathrm{mHz}$, respectively.

Fig. 2c). The relationship of $\delta \tau$ with $B_{\text {tot }}$ with all filters is consistent with predominately near-surface perturbations but does not rule out subsurface perturbations, which may very well correlate with surface flux.

\subsection{Consistency with a Deep Sound-Speed Proxy}

The mean travel-time perturbations $\delta \tau$ from each filter and frequency combination were averaged over several sunspot umbrae in the observed field. The fractional travel-time perturbations $\left(\delta \tau / \tau_{g}\right.$, where $\tau_{g}$ is the mean group travel-time of the modes within the filter) are shown in Figure $3 a$ as functions of the phase speed $w$. There is a general decrease of the strength of the umbral average of $\delta \tau / \tau_{g}$ with increasing $w$ for pupils D$\mathrm{J}$; however, the systematic frequency variations are clearly visible over this trend. We do not attempt to directly model the travel-time observations obtained here. Instead, we compare a subset of the observations, the umbral averages, with expectations from a proxy sound-speed perturbation based on previous travel-time inversions. The solid colored lines indicate the fractional travel-time perturbations expected for rays originating in the center of a cylindrical sound-speed perturbation that is horizontally uniform out to a $15 \mathrm{Mm}$ radius and that has a specified depth dependence. This dependence was selected by trial and error to provide a reasonable representation of the data at $3 \mathrm{mHz}$ while preserving the general features of recent time-distance inversions (e.g., Kosovichev et al. 2000; Jensen 2003; Couvidat et al. 2006). Our proxy is defined by 


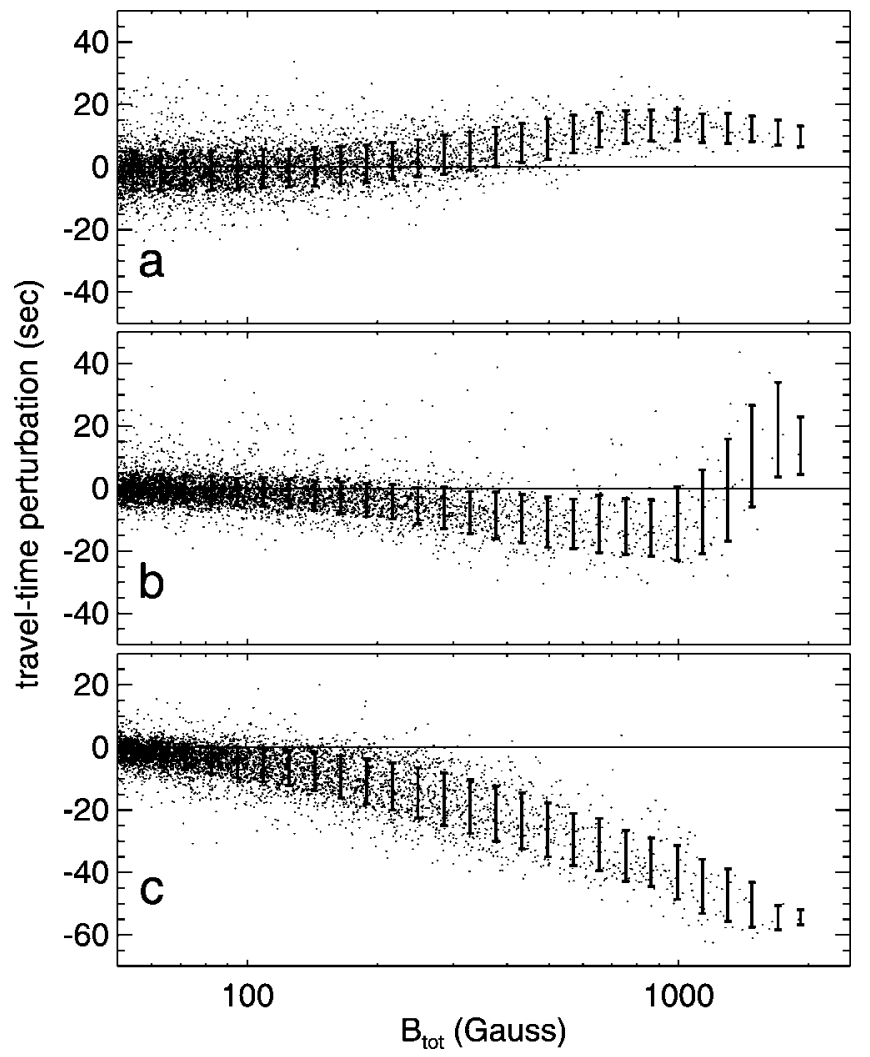

FIG. 2.-Scatter plots of $\delta \tau$ against the total magnetic flux density $B_{\text {tot }}$ (estimated from the MDI line-of-sight magnetogram assuming the magnetic field is the gradient of a potential). For clarity, only 1 out of 15 points is plotted. The vertical bars indicate the range of \pm 1 standard deviation about the mean over bins equally spaced over the logarithm of the flux density. The three cases shown are representative samples of the types of variations observed: (a) shows the results for filter A at $\nu_{0}=5 \mathrm{mHz},(b)$ shows filter $\mathrm{C}$ at $3 \mathrm{mHz}$, and (c) shows filter $\mathrm{E}$ at $4 \mathrm{mHz}$.

the difference, $\delta c$, between the sound speed inside the cylinder and a background sound speed, $c$, from a solar model (Christensen-Dalsgaard et al. 1996), where $\delta c=1.9 \exp [-(z / 20$ $\left.\mathrm{Mm})^{2}\right]-2.3 \exp \left[-(z / 5 \mathrm{Mm})^{2}\right] \mathrm{km} \mathrm{s}^{-1}$, and $z$ is the depth. This gives a fractional perturbation $\delta c / c=-0.06$ at the photosphere and a positive perturbation below depths of $2 \mathrm{Mm}$, with a maximum $\delta c / c=0.08$ at $5 \mathrm{Mm}$. Mean travel-time perturbations were computed in the ray approximation (Kosovichev \& Duvall 1997) using frequency-dependent ray paths (Barnes \& Cally 2001). A recent comparison between rayapproximation- and Born-approximation-based inversions for sound speed (Couvidat et al. 2006) suggests that the ray approximation may be sufficient to treat sound-speed perturbations of the type considered here, although this has not been established over the entire frequency range employed in this study. It is clear that the predictions of the proxy do not match the observed travel times for frequencies other than $3 \mathrm{mHz}$. For pupils E-J, the $5 \mathrm{mHz}$ data are consistently underestimated by the model by about a half, while the $2 \mathrm{mHz}$ data are overestimated by about a factor of 2 . It is likely that the agreement of the proxy predictions and the observations at any given frequency could be improved with a different choice of the sound-speed variation. However, no simple adjustments of the proxy at depths comparable to the lower turning points of the modes can explain the large systematic frequency variations that are observed.
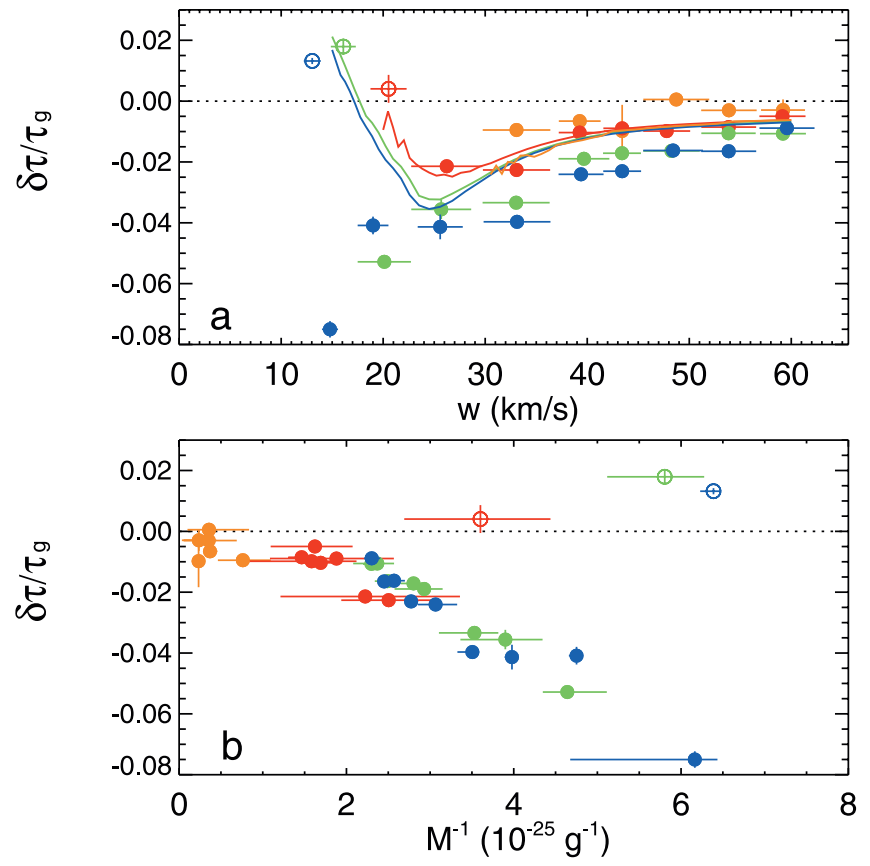

FIG. 3.-(a) Mean travel-time perturbations, averaged over all sunspot umbrae in the four spot groups observed, divided by the group travel times as functions of the phase speed $w$, where the orange, red, green, and blue symbols indicate results for $\nu_{0}=2,3,4$, and $5 \mathrm{mHz}$, respectively. The open circles indicate the filter and frequency combinations which are dominated by the $p_{1}$-mode. Horizontal bars indicate the range of $w$ present within the filter and frequency bandpass, and the vertical bars indicate the standard deviation of the mean. The colored lines indicate the predicted values computed from a sound-speed proxy (see text), where the colors indicate the same $\nu_{0}$ as the observations. (b) Fractional travel-time perturbations as a function of the inverse of the mean mode mass.

\section{DISCUSSION}

The past decade has seen major advances in modeling and interpreting the travel times measured in and around sunspots. It appears, however, that the strong frequency variation of the measured travel times presented here cannot be explained using standard assumptions, i.e., standard ray-approximation-based forward modeling applied to sound-speed models that are typical of published 3D inversion results. One possible explanation for the discrepancy between the models and the data is that the models do not include surface effects. It is premature to estimate how inferences about subsurface structure will likely change when these effects are incorporated into modeling efforts, and we do not attempt such modeling here. However, a rough assessment of the importance of surface effects may be made by examining the dependence of observed travel-time perturbations with mode mass. Perturbation theory applied to global $p$-mode frequencies (e.g., Christensen-Dalsgaard et al. 1988; Libbrecht \& Woodard 1990) predicts that the contribution to the fractional frequency perturbations due to surface effects is some function of frequency divided by the mode mass $M_{n l}$, which is defined as the ratio of mode kinetic energy to the square of the velocity evaluated at a height near the photosphere. In local helioseismology we expect a similar functional form for the travel-time perturbation resulting from a phase shift in an unresolved layer near the surface. This follows from the asymptotic equivalence of a fractional frequency perturbation $\delta \nu_{n l} / \nu_{n l}$ of a $p$-mode with the perturbation $\delta \tau / \tau_{g}$ experienced by a wave packet consisting of a range of modes about 
the same $n$ and $l$. In the limit that the contributions of surface effects to the travel-time perturbations are substantially greater than structural contributions, we might expect the observed perturbations to exhibit frequency and mode-mass dependences consistent with this relation.

Figure $3 b$ shows $\delta \tau / \tau_{g}$ for the same umbral averages shown in Figure $3 a$, as a function of the inverse of the mode mass, $\left\langle M_{n l}\right\rangle^{-1}$ averaged over the range of radial order $n$ and angular degree $l$ present in the filter and frequency bandpass The mode mass $M_{n l}$ is evaluated at $50 \mathrm{~km}$ above the photosphere (the choice of this height does not substantially affect the observed trend). There is a striking, nearly linear, correlation (with negative slope) of the umbral travel-time perturbations with $\left\langle M_{n l}\right\rangle^{-1}$ for modes with $n>1$, although there is a hint of a separate, linear relation (with a positive slope) for the $p_{1}$-modes as well. A somewhat tighter linear relation can be achieved by multiplying the abscissa by a slightly decreasing function of frequency. The nonlinearity of the trend may also reflect the failure of $\left\langle M_{n l}\right\rangle^{-1}$ to adequately describe the physics of the surface perturbation, the presence of deeper structural contributions to $\delta \tau$, or systematic errors in the observations. Nevertheless, we suggest that the general trends shown in Figures $3 a$ and $3 b$ are sufficient to demonstrate that surface effects may contribute significantly to the observed travel-time perturbations. We wish to emphasize that the findings presented here do not necessarily demonstrate that $p$-mode traveltime perturbations are entirely superficial in origin.

Similar trends to those shown in Figure 3 are also exhibited by travel-time perturbations averaged over pixels in both the penumbrae and in plages. Two of the three $p_{1}$-dominated measurements switch sign in both penumbrae and plage (see $\S \S 3.1$ and 3.2) but continue to exhibit smaller (absolute) perturbations than compared with other measurements with either a similar $w$ or mode mass. In other words, the $p_{1}$ measurements consistently show anomalous (outlier) behavior in essentially all representations of the data considered. An important outstanding question is whether this anomaly reflects some particular physical property of $p_{1}$-modes or systematic uncertainties (e.g., due to the use of very small annuli within sunspots).

An important caveat to our conclusions is that the accuracy of the ray approximation (or, for that matter, the Born approximation) has not been assessed over the frequency range employed in this study. However, what should be evident from the results shown here is the importance of successfully modeling both the phase-speed and frequency dependence of the observations (and of understanding the anomalous behavior of the $p_{1}$-modes), in as much as is practical, in order to have confidence in the applicability of the models to the solar interior. In conclusion, these findings strongly suggest that significant improvements in the modeling of the subsurface structure of sunspots and magnetic regions in the Sun with helioseismology are likely to be made with the consideration and inclusion of surface effects due to magnetic fields.

D. C. B. gratefully acknowledges advice from and discussions with J. Zhao. We appreciate helpful comments from P. Cally, T. Duvall, Jr., A. Kosovichev, C. Lindsey, M. Woodard, J. Zhao, and the anonymous referees. D. C. B. gives a special thanks to Lisa Yulan Braun for putting everything in perspective. This work is supported by funding from the National Aeronautics and Space Administration, through the Solar and Heliospheric Physics (SR\&T) and Living with a Star (TR\&T) programs, and the National Science Foundation, through the Stellar Astronomy and Astrophysics program.

\section{REFERENCES}

Abdelatif, T. E., Lites, B. W., \& Thomas, J. H. 1986, ApJ, 311, 1015 Barnes, G., \& Cally, P. S. 2001, Publ. Astron. Soc. Australia, 18, 243

Basu, S., Antia, H. M., \& Bogart, R. S. 2004, ApJ, 610, 1157

Braun, D. C. 1997, ApJ, 487, 447

Braun, D. C., Duvall, T. L., Jr., \& LaBonte, B. J. 1987, ApJ, 319, L27

Braun, D. C., \& Lindsey, C. 2000, Sol. Phys., 192, 307

Cally, P. S., Crouch, A. D., \& Braun, D. C. 2003, MNRAS, 346, 381

Chou, D.-Y., Sun, M.-T., \& Chang, H.-K. 2000, ApJ, 532, 622

Christensen-Dalsgaard, J., Gough, D. O., \& Perez Hernandez, F. 1988, MNRAS, 235, 875

Christensen-Dalsgaard, J., et al. 1996, Science, 272, 1286

Couvidat, S., Birch, A. C., \& Kosovichev, A. G. 2006, ApJ, 640, 516

Couvidat, S., Birch, A. C., Kosovichev, A. G., \& Zhao, J. 2004, ApJ, 607, 554

Crouch, A. D., Cally, P. S., Charbonneau, P., Braun, D. C., \& Desjardins, M. 2005, MNRAS, 363, 1188

Duvall, T. L., Jr., D’Silva, S., Jefferies, S. M., Harvey, J. W., \& Schou, J. 1996, Nature, 379, 235

Fan, Y., Braun, D. C., \& Chou, D.-Y. 1995, ApJ, 451, 877

Hughes, S. J., Rajaguru, S. P., \& Thompson, M. J. 2005, ApJ, 627, 1040

Jensen, J. M. 2003, in Local and Global Helioseismology: The Present and Future, ed. H. Sawaya-Lacoste (Noordwijk, ESA), 315
Jensen, J. M., Duvall, T. L., Jr., Jacobsen, B. H., \& Christensen-Dalsgaard, J. 2001, ApJ, 553, L193

Kosovichev, A. G., \& Duvall, T. L., Jr. 1997, in SCORe '96: Solar Convection and Oscillations and Their Relationship, ed. F. P. Pijpers, J. ChristensenDalsgaard, \& C. S. Rosenthal (Dordrecht: Kluwer), 241

Kosovichev, A. G., Duvall, T. L., Jr., \& Scherrer, P. H. 2000, Sol. Phys., 192, 159

Libbrecht, K. G., \& Woodard, M. F. 1990, in Lecture Notes in Physics, 367, Progress of Seismology of the Sun and Stars (Berlin: Springer), 145

Lindsey, C., \& Braun, D. C. 1997, ApJ, 485, 895

2000, Sol. Phys., 192, 261

2004, in Helio- and Asteroseismology: Towards a Golden Future, ed.

D. Danesy (ESA SP-559: SOHO 14; Noordwijk: ESA), 552 2005a, ApJ, 620, 1107 2005b, ApJ, 620, 1118

Rajaguru, S. P., Birch, A. C., Duvall, T. L., Jr., Thompson, M. J., \& Zhao, J. 2006, ApJ, 646, 543

Scherrer, P. H., et al. 1995, Sol. Phys., 162, 129

Schunker, H., Braun, D. C., Cally, P. S., \& Lindsey, C. 2005, ApJ, 621, L149

Thomas, J. H., Cram, L. E., \& Nye, A. H. 1982, Nature, 297, 485

Zhao, J., \& Kosovichev, A. G. 2006, ApJ, 643, 1317

Zhao, J., \& Kosovichev, A. G., \& Duvall, T. L., Jr. 2001, ApJ, 557, 384 